\title{
Foot Ulcers: A Different Technique to Total Contact Casting for Healing Chronic Foot Ulcers
}

\author{
Maria Shaw \\ Orthopaedic Clinical Nurse Specialist - Lead, Orthopaedic \\ Outpatients Department, Mid Central DHB, New Zealand.
}

*Corresponding author

Maria Shaw, Orthopaedic Clinical Nurse Specialist - Lead, Orthopaedic Outpatients Department, Mid Central DHB, New Zealand, E-Mail: mariashaw2001@yahoo.com.au.

Submitted: 28 Sep 2017; Accepted: 04 Oct 2017; Published: 09 Oct 2017

\section{Introduction}

Total Contact Cast (TCC) is considered the gold standard method for healing diabetic foot ulcers (DFU) [1]. Chronic foot ulcers are a growing concern worldwide. Evidence-based research suggests that TCC is the best method to offload the plantar foot by adequately redistributing plantar pressures related to body mass while still maintaining patient mobility.

Although the excellent healing success of a TCC is considered the gold standard for healing diabetic foot ulcers (DFU), using alternative methods to achieve healing is sometimes needed [1].

The main types of ulcers seen in the lower extremities are venous and neuropathic.

In 2015 the prevalence data from the international Diabetes Federation estimated that 9.1 million people with diabetes will develop foot ulcers worldwide [2].

This article aims to rationalize the introduction of an alternative technique to offloading chronic foot ulcers from TCC and to aid healthcare professionals overcome the difficulties and fear of TCC and to develop the skill to applying this uncomplicated method of soft offloading bandage, and showing the results of healing chronic foot ulcers within 3 to 5 months using this technique.

Within the Orthopaedic service we see mostly neuropathic ulcers and Charcot foot ulcer. Charcot foot is a consequence of various peripheral neuropathies, and diabetic neuropathy is the most common reason [3].

Charcot neuropathic osteoarthropathy, commonly referred to as the Charcot foot, is a condition affecting the soft tissue, bones and joints of the foot and the ankle, characterised by inflammation in the early stage. The Charcot foot is believed to be a consequence of various peripheral neuropathies and diabetic neuropathy has become the most common etiology. Complications resulting from several component factors can be present such as diabetes, sensory-motor neuropathy, autonomic neuropathy, trauma, and metabolic abnormalities of bone results in an acute localized inflammatory condition that may lead to varying degrees of bone destruction, subluxation, dislocation, and deformity. The deformity associated with this condition is known as mid foot collapse, described as a "rocker-bottom" foot [4]. These patients are referred to the Orthopaedic service because of bone involvement and consequence formation of an ulcer.

Neuropathy causes the loss of pain sensation to the foot which may lead to the patient exerting a lot of pressure at one spot under the foot when walking, causing a build-up of callous at the site but would be without causing an awareness of discomfort. This may cause an open wound which in turn forms the ulcer.This callous edge may also cause increased plantar pressure and is the leading cause of ulceration.

The greatest amount of pressure placed on a foot ulcer is at the wound edges and is often referred to as the leading edge or edge effect [5].

Because there is a natural offloading of the central wound cavity, the plantar foot pressures intensify at the leading edge of the wound.

The goal of TCP relief is balancing the forces on the plantar foot. By redistributing the forces, pressure at the ulcerative site will be offloaded.

Keep in mind that TCP relief does not necessarily mean Total Contact Cast as many devices can redistribute plantar foot pressure.

The first and most important offloading technique that needs addressing is debriding the skin surrounding the wound edge or the "edge effect" which results in tissue damage from both shearing and vertical stress. Therefore, effective offloading must take both of these destructive forces into account when determining which technique to use to offload the ulcer [6].

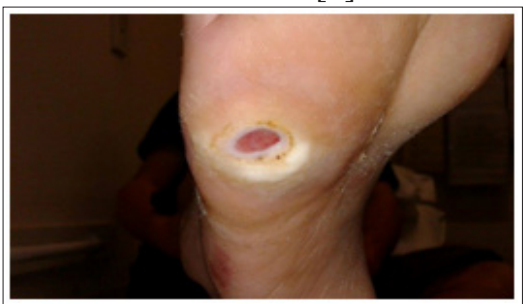

Debriding the ulcer edge 
The orthopaedic service within our DHB has had an increase in the number of referrals for TCC for foot ulcers. These referrals have been mainly from the surgical service and from the community podiatrist.

Their aim was to offload the ulcer with TCC, as they had tried numerous ways to heal the ulcers with little success and these are now chronic ulcers as the patients have had them over a number of months or a year. Without adequate resources available to manage the increased numbers being referred and just a limited number of staff with the expert knowledge who could apply a TCC, we had to find an alternative method that was effective and easy to apply to this patient group without have the need of a trained plaster technician or medical staff.

Total Contact Cast can be either Plaster of Paris or Total Synthetic cast. In order to avoid new skin breakdown and ensure patient safety, an experienced cast technician or physician should apply the TCC.These casts have to be removed by a plaster saw. That is another issue as most staff will not use the saw if not trained in the safe use of them.

While most studies regarding TCC report low rates of complications, they are not without contraindications-

- TCC should not be used on patients with deep abscess, gangrene, osteomyelitis, chronic venous stasis ulcers or severe peripheral arterial disease.

- They should be used with caution in patients who have an unsteady gait or with noncompliant patients who routinely miss scheduled appointments.

- $\quad$ Patients can also develop skin break down around the foot and ankle area depending on sensitivity of the skin, and length of time in the cast. Ulcers improve with this method but can take over a year or more to heal especially the Chronic Ulcer.

- The numerous dressing and cast changes have a substantial cost to the DHBs and a significant impact on a patient's quality of life

- $\quad$ TCC for ulcers that have heavy exudate need a window cut out round the site of the ulcer to expose the wound for easy access for wound cares and review of the progress of the ulcer.

Looking at different methods to offload and protect the surrounding skin was challenging. I had attended a Plaster Workshop and observed a plaster technician applying an alternative TCC. Plaster stocking soffban was used and then two or three rolls of synthetic cast Very good results occurred with this. However I felt that this would be quite expensive as the cast would need changing often, depending on exudate and may require a plaster saw to remove depending on the synthetic cast used.

By changing the method of offloading to a soft offloading bandage with no synthetic cast over the top of the dressing and just applying crepe bandages we have reduced the need for a window to be cut as previously needed in a TCC and reduced the incidence of complications of skin breakdown. Using the Soft offloading Bandage we have maintained a high healing rate in comparison to that of TCC. Patients have also remained mobile and have found the bandage comfortable. The bandage may need to be changed twice a week depending on exudate at the start of the process, once this is under control and the amount reduced then the bandage can be changed once a week.

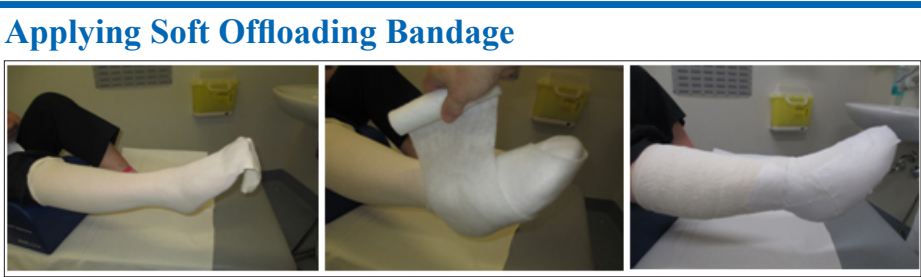

Plaster stockinet Soft synthetic wool x 4

Crepe Bandage x 2

To apply a Soft Offloading Bandage:

- $\quad$ First make sure the callous edges have been debrided, dress the ulcer with appropriate wound care produce, felt around the ulcer for extra protection and offloading the area.

- Apply the plaster stockinet; incorporating the toes

- Apply at least 4 large rolls of soft synthetic wool bandage.

- Apply two crepe bandages and tape to secure bandage.

- Then use either a moonboot or Orthotic shoe for patient to mobilise with

- This technique has also been used on pressure ulcers on the lateral or medial malleolus caused by pressure from shoes or orthotics.

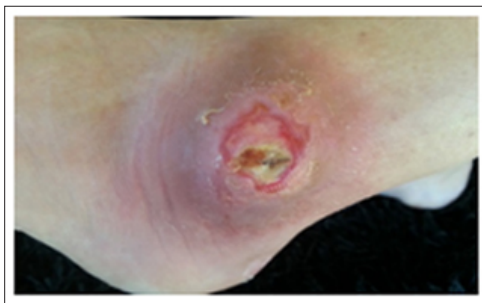

Malleolus pressure ulcer

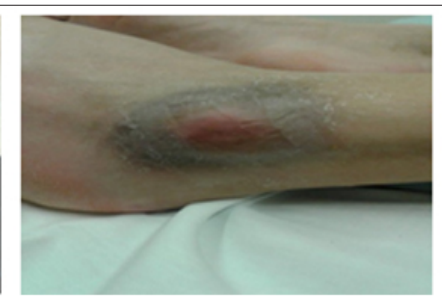

Healed within 4 to 5 months
It is cost effective in comparison to TCC when numerous dressing changes are required.

It does not require experienced plaster technicians or experienced physicians to apply. It is easy to put on making dressing changes more convenient. It is also easy to remove if the patient is having issues with the wound or become unwell and the Doctor needs to review the wound quickly without the use of a plaster saw.

If you have a non-compliant patient that miss appointments the bandage can be easily removed by the patient if needed or if left on longer than anticipated generally doesn't cause any harm to the skin except in some cases some maceration depending on amount of exudate?

I have trialled the Soft Offloading Bandage on 10 patients within the department

- 1 removed from the study due to exacerbation of their comorbidities,

- $\quad 1$ osteomyelitis and were hospitalized.

- 1 removed as was not tolerating bandages on her leg and refused all suggestions for offloading.

- 5 patients with foot ulcers and pressure sore

- 1 lateral malleolus pressure sore

- 1 bilateral malleolus pressure sores

- All healed within two to five months 


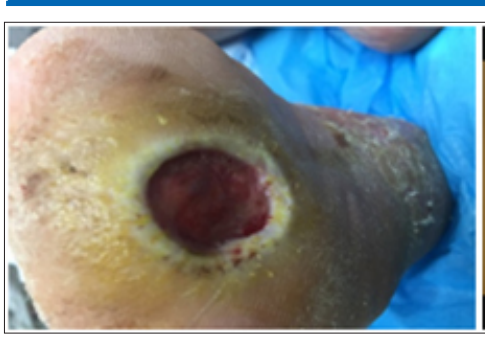

Chronic diabetic foot ulcer

Healed within $3 \mathrm{mths}$

Not included in this study are the acute sores which healed within a much shorter time frame.

\section{Conclusion}

By simply altering the offloading technique, regular debriding of the ulcer edges and keeping the foot warm,faster healing rates for chronic ulcers resulted. Although not suitable for all patients there was a significant reduction in the time frame for healing chronic ulcers. This had a positive impact on the patient's quality of life and a marked reduction in costs to the DHB.

This technique can be taught to all health professionals in a very short period of time as there is no complicated technique involved. These patients benefit from a Multi-Disciplinary approach. The biggest obstacle is making sure the ulcer is debrided regularly. Working with a podiatrist is beneficial, as they look after that aspect of the wound. Once the callous is controlled it is recommended that the patient obtains an electric hand held battery operated callous remover. This gives them control of their foot care and the callous can be controlled at home. It is important to educate patients on how to look after their feet by regularly checking for sores, cuts or discolouration. Not all patients will have access to a podiatrist or will be able to afford an appointment to see one. Finance is an issue for majority of our patients. A number of patients have used these devices and have reduced the callous build up around the healed ulcer site which I have found reduces the problems with recurring ulcers, however if they do not have appropriate foot wear with properly fitted offloading orthotics then the ulcers will break down again.

It is important to realise that once these ulcers are healed the patients' need to have adequate foot wear with a moulded orthotic inserted in the shoe to continue with offloading the pressure points associated with the ulcer.

More work is needed to look at appropriate and affordable foot wear and better education for diabetics in foot care management. Until we get that right, these ulcers will continue to a problem.

Not all patients are going to benefit from this Offloading technique. Patients will need to be risk assessed for possible application of Offloading techniques including TCC. They need to understand and be compliant with the process to ensure a good outcome [7-25].

\section{References}

1. Diabetic Foot Ulcers and Their Recurrence

2. Armstrong DG, Boulton AJ, Sicco A (2017) Diabetic Foot Ulcers and Their RecurrenceNew England Journal of Medicine 376: 2367-2375.

3. Rogers LC (2011) The Charcot foot in diabetes. Diabetes Care 34: $2123-2129$.
4. Jeffcoate WJ, Game F, Cavanagh PR (2005) The role of proinflammatory cytokines in the cause of neuropathic osteoarthropathy (acute Charcot foot) in diabetes. Lancet 366: 2058-2061.

5. Armstrong DG, Nguyen HC, Lavery LA, Van Schie CH, Boulton AJ, et al. (2001) Offloading the diabetic foot wound. Diabetes Care 24: 1019-1022.

6. Edwards J, Stapley S (2010) Debridement of diabetic foot ulcers. Cochrane Database Syst Rev CD003556.

7. Armstrong DG, Athanasiou KA (1998) The edge effect: how and why wounds grow in size and depth. Clinical Podiatry Med Sur 105-108.

8. Armstrong DG, Lavery LA, et al. (2004) It is not what you put on, but what you take off: techniques for debriding and offloading the diabetic foot wound. Clin Infect Dis 39: S92-99.

9. Boulton AJ, Vileikyte L, Ragnarson-Tennvall G, Apelqvist J (2005) The global burden of diabetic foot disease. Lancet 366: 1719-1724.

10. Espensen E (2007) Assessing Debridement Options For Diabetic Wounds. Podiatry Today 20.

11. Fard AS, Esmaelzadeh M, Larijani B (2007) Assessment and treatment of diabetic foot ulcer. Int J Clin Pract 61: 1931-1938.

12. Fife C, Carter M, Walker D (2010) Why is it so hard to do the right thing in wound care? Wound Repair \& Regeneration [serial online] 18: 154-158.

13. JAMES A. BIRKE, M.S.: DAVIS S. SIMS, JR., M.A. ; WILLIAM L. BUFORD, PH.D. Department of Physical Therapy and Rehabilitation Research, National Hansen's Disease Center, Carville, Louisiana 70721 walkingcasts: effect on plantar foot pressures. Journal of Rehabilitation Research and Development 22: 3BPR 10-42 Pages 18-22.

14. Joakola E, Weber A (2014) Current concepts in total contact casting for DFU's. Podiatry today 27.

15. Leibner ED, Brodsky JW, Pollo FE, Baum BS, Edmonds BW (2006) Unloading mechanism in the total contact cast. Foot Ankle Int 27: 281-285.

16. Lewis J, Lipp A (2013) Pressure-relieving interventions for treating diabetic foot ulcers. Cochrane Database of Systematic Reviews, Art. No.:CD002302.

17. Lindy Begg L, Burns J, Fletcher J, McLaughlin P, Vicaretti (2016) Total contact cast wall load in patients with a plantar forefoot ulcer and diabetes. Journal of Foot and Ankle Research 9: 2.

18. Myerson M, Papa J, Eaton K, Wilson K (1992) The total contact cast for management of neuropathic plantar ulceration of the foot. JBJS (Am) 74: 261-269. Nick Martin, DPM, Tim Oldani, DPM, and Matthew J. Claxton, DPM. Podiatry today Issue Number 9 Volume 18 September 2005.

19. Petre M, Tokar P, Kostar D, Cavanagh PR (2005) Revisiting the total contact cast: maximizing off-loading by wound isolation. Diabetes Care 28: 929-930.

20. Sawacha Z, Gabriella G, Cristoferi G, Guiotto A, Avogaro A, et al. (2009) Diabetic gait and posture abnormalities: a biomechanical investigation through three dimensional gait analysis. Clin Biomech (Bristol, Avon) 24: 722-728.

21. Seaman $S$ (2005) The role of the nurse specialist in the care of patients with diabetic foot ulcers. Foot Ankle Int 26:19-26.

22. Shishir Shah, DO Clinical and Economic Benefits of Healing Diabetic Foot Ulcers With a Rigid Total Contact Cast.

23. Shau S (2012) Do Clinical and Economic Benefits of Healing Diabetic Foot Ulcers With Rigid Total Contact Cast. Wounds 
24: $152-159$.

24. Snyder RJ, Hanft JR (2009) Diabetic foot ulcers-effects on QOL, costs, and mortality and the role of standard wound care and advanced-care therapies. Ostomy Wound Manage 55: 28-38.
25. Wu SC, Jensen JL, Weber AK, Robinson DE, Armstrong DG (2008) Use of pressure offloading devices in diabetic foot ulcers: so we practice what we preach? Diabetes Care 31: 2118-2119.

Copyright: (2017 Maria Shaw. This is an open-access article distributed under the terms of the Creative Commons Attribution License, which permits unrestricted use, distribution, and reproduction in any medium, provided the original author and source are credited. 